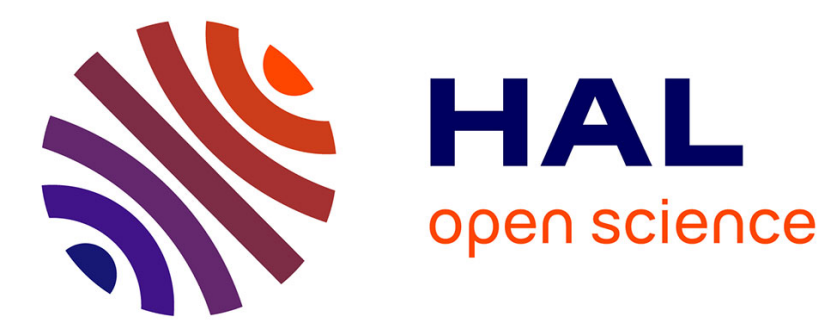

\title{
Présentation de l'ouvrage Action publique et légitimités professionnelles
}

Dominique Bessières

\section{To cite this version:}

Dominique Bessières. Présentation de l'ouvrage Action publique et légitimités professionnelles. Formation Emploi. Revue française de sciences sociales, 2009, 106, pp.93-96. 10.4000/formationemploi.1963 . halshs-01343573

\section{HAL Id: halshs-01343573 \\ https://shs.hal.science/halshs-01343573}

Submitted on 8 Jul 2016

HAL is a multi-disciplinary open access archive for the deposit and dissemination of scientific research documents, whether they are published or not. The documents may come from teaching and research institutions in France or abroad, or from public or private research centers.
L'archive ouverte pluridisciplinaire HAL, est destinée au dépôt et à la diffusion de documents scientifiques de niveau recherche, publiés ou non, émanant des établissements d'enseignement et de recherche français ou étrangers, des laboratoires publics ou privés. 
106 (avril-juin 2009)

Pêle-mêle

Dominique Bessières

\title{
Présentation de l'ouvrage Action publique et légitimités professionnelles
}

\begin{abstract}
Avertissement
Le contenu de ce site relève de la législation française sur la propriété intellectuelle et est la propriété exclusive de l'éditeur.

Les œuvres figurant sur ce site peuvent être consultées et reproduites sur un support papier ou numérique sous réserve qu'elles soient strictement réservées à un usage soit personnel, soit scientifique ou pédagogique excluant toute exploitation commerciale. La reproduction devra obligatoirement mentionner l'éditeur, le nom de la revue, l'auteur et la référence du document.

Toute autre reproduction est interdite sauf accord préalable de l'éditeur, en dehors des cas prévus par la législation en vigueur en France.
\end{abstract}

revues.org

Revues.org est un portail de revues en sciences humaines et sociales développé par le Cléo, Centre pour l'édition électronique ouverte (CNRS, EHESS, UP, UAPV).

Référence électronique

Dominique Bessières, «Présentation de l'ouvrage Action publique et légitimités professionnelles », Formation emploi [En ligne], 106 | avril-juin 2009, mis en ligne le 19 novembre 2009, consulté le 14 janvier 2013. URL : http:// formationemploi.revues.org/1963

Éditeur : La documentation française http://formationemploi.revues.org

http://www.revues.org

Document accessible en ligne sur : http://formationemploi.revues.org/1963

Ce document est le fac-similé de l'édition papier.

( ) Tous droits réservés 


\section{Note
delecture}

\section{Présentation de l'ouvrage "Action publique et légitimités professionnelles»}

\section{Dominique Bessières*}

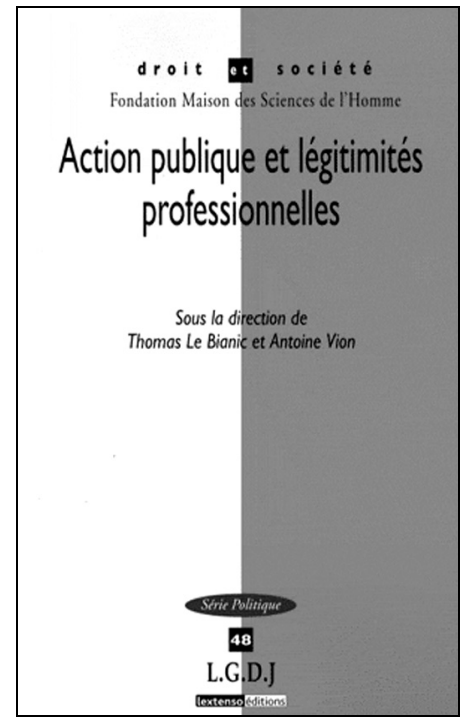

Un des intérêts premiers de l'ouvrage est de croiser deux disciplines, la sociologie des professions d'une part, la science politique des politiques publiques, d'autre part. De ce point de vue, l'appartenance disciplinaire des deux responsables de l'ouvrage illustre cette perspective d'interdisciplinarité pour appréhender la réalité des mutations à l'œuvre. D'emblée, le parti pris est de présenter un regard d'actualité de nature à jeter un pont entre des grilles d'analyse disciplinaires modernisées par une attention aux questions des légitimités professionnelles et aux conditions de l'action publique. Les seize contributions montrent " l'interpénétration de l'État et des professions ».

L'intérêt de l'ouvrage porte ainsi sur une dimension assez originale de l'influence croissante de stratégies professionnelles dans l'action publique, à double titre :

- Il se focalise sur une observation bien étayée par un large échantillon d'acteurs professionnels (direc- teurs de la PJJ - protection judiciaire de la jeunesse, médecins des réseaux de santé, médecins inspecteurs de santé publique, inspecteurs du travail, pédiatres des hôpitaux, agents d'accueil et conservateurs des musées, conseillers principaux d'éducation, ingénieurs des ponts et chaussées, aménageurs et associations...) relevant d'une action d'État de service public, qu'elle soit le fait de professions établies ou émergentes. Les cas étudiés appartiennent au contexte

* Dominique Bessières est maître de conférences à I'université de Reims Champagne - Ardenne et à l'Institut d'études politiques de Lille, 71 e section Sciences de l'information et de la communication. II a publié récemment : « Le modèle des professions, enjeu de légitimation étatique pour la communication publique : simulacre ou effet de réalité ", Colloque international, ISA International Sociological Association / Association internationale de sociologie, 5e Conférence intermédiaire du Comité de recherche 52 "Sociologie des groupes professionnels ", Le modèle des professions en question : apports et limites du professionnalisme, Oslo University College, Norvège, $12-13$ septembre 2008. "L'impact des TIC dans les IUFM : de nouveaux usages sous influence managériale ou une injonction paradoxale? ", Colloque international, Association internationale des sociologues de langue française, XVIIIle Congrès - Istanbul, Turquie, GT 13 « Sociologie de la Communication », 7-1 1 juillet 2008. 
étatique français. De ce point de vue, la démarche revêt un certain particularisme en comparaison des initiateurs des courants d'analyse de la sociologie des professions. Ceux-ci, majoritairement anglo-saxons, privilégient des terrains d'observation appartenant au secteur privé.

- Il pointe des modalités assez fines et peu étudiées de la mise en œuvre de l'action publique par l'accent délibérément mis sur les catégories d'acteurs publics internes : l'importance du développement adaptatif de professionnalités. L'analyse de la mise en œuvre des politiques publiques est ainsi déplacée par rapport aux classiques négociations représentants politiques/administrations, et focalisée au plus près de certains terrains.

L'organisation de l'ouvrage est d'actualité dans la mesure où l'émergence de l'enjeu de l'affirmation de légitimités professionnelles au sein de l'État est expliquée. Ceci est assez nouveau parce qu'en opposition avec le schéma, classique, d'organisation bureaucratique étatique. Plus fondamentalement, une voie d'explication à cette nouvelle source de légitimité est évoquée à différents passages de l'ouvrage, en particulier en conclusion : les changements contextuels. L'influence des mutations issues de la construction européenne privilégiant l'hybridation des formes d'expertise (gouverner par les instruments), la montée en puissance des formes de concertations et de débats publics participatifs et délibératifs (développement de la notion d'usager, chap. 6 et 9), semblent de nature à engendrer une plus grande procéduralisation. Ce type de logique d'adaptation de l'action étatique semble fécond pour l'appréhension globale du développement des légitimités professionnelles.

Enfin, l'ouvrage s'avère très pertinent dans l'articulation systématique des analyses des terrains précisément contextualisées et des synthèses plus théoriques présentes dans l'introduction et la conclusion générale, mais aussi dans les introductions des quatre parties, avec une quatrième partie théorique. On évite ainsi les insuffisances du manque de généralité des monographies et d'une trop grande abstraction déconnectée des réalités de terrain. Enfin, la volonté d'appréhender les évolutions sous tous les angles est étayée, de façon assez systématique, par un enrichissement d'approches micro, méso et macro sociologiques. De sorte que le matériau heuristique est riche.

Ces remarques générales étant faites, l'objet affiché de l'ouvrage est d'étudier quels professionnels sont recherchés pour répondre aux problèmes de la société contemporaine. Les auteurs notent l'émergence récente des métiers flous de médiateurs liés à l'élargissement du système de décision politique étatique avec l'Europe et la décentralisation. Elle se traduit par le développement de la gouvernance et du pilotage de l'action publique, du nouveau management des ressources humaines. On observe deux évolutions : l'action publique multi-organisationnelle et la fin d'une administration de production privilégiant une logique de réalisation en dynamique et non plus simplement de résultat par rapport à ces normes. Les statuts rigides corporatistes sont ainsi amenés à être assouplis, pour substituer aux solutions standardisées la contingence adaptée au terrain.

La question de la légitimité professionnelle et de ses évolutions est un fil rouge. Elle se fonde sur le partage d'une croyance support d'une domination (Weber), d'une autorité scientifique support d'une autonomie (Friedson). Historiquement et spatialement datée, cette question se repose à propos d'un nouveau type de légitimité visé dans les différents cas présentés dans l'ouvrage entre autonomie et contrôle, avec le nouveau management public (new public management). La plupart des chapitres abordent cette thématique vis-à-vis des usagers (chap. 5, 8,12 ), des valeurs sociétales (chap. 1, 4, 6, 7), des mécanismes de représentation (chap. 2, 3, 11).

L'ouvrage est divisé en quatre parties qui rendent compte de l'obsolescence des schémas d'organisation professionnels pyramidaux.

\section{Partie I - Les recompositions de la coordination bureaucratique}

Cette partie s'intéresse à l'influence du nouveau management public sur des professions à forte identité (chap. 1: éducateurs avec l'importance des attentes de l'État; chap. 2 : médecins vis-à-vis de l'influence managériale ; chap. 3 : médecins inspecteurs de santé publique dépendant de l'émergence d'un référentiel ad hoc; chap. 4: inspecteurs du travail à l'abandon dans un contrôle public faible). Il 
ressort que l'action publique est professionnelle et politique (micro et macro). Les cadres, en augmentation, manifestent une plus grande réactivité vis-à-vis du terrain, générant chez eux et dans l'organisation des tensions identitaires et culturelles. On note aussi un développement des fonctions de coordination et d'interface liées à l'adoption de méthodes de travail moins cloisonnées. Consécutivement, on est en présence d'un développement des domaines de négociation. La rationalité managériale s'exprime donc dans tous les cas, au point que les outils de gestion apparaissent constitutifs de l'action publique.

\section{Partie II - Le rôle des usagers dans l'évaluation des pratiques et la relégitimation de l'action publique}

Cette partie traite des professions émergentes avec le développement des fonctions d'accueil et d'accompagnement (chap. 5 : personnel soignant de pédiatrie et les nouveaux droits des usagers; chap. 6: les agents d'accueil des musées revalorisés dans un souci de démocratisation culturelle; chap. 7: les Conseillers Principaux d'Éducation également revalorisés entre les enseignants et les chefs d'établissement; chap. 8 : les policiers sur la voie publique). Les usagers sont au cœur des actions de modernisation de l'État, avec en fond l'individualisation des services publics. Ces évolutions du travail sont génératrices de stress et de résistances dans la mesure où les professionnels doivent articuler les anciennes références de bonne pratique professionnelle entre pairs avec la recherche de la satisfaction des publics.

\section{Partie III - Des savoirs canoniques aux savoirs composites : les modes opératoires à l'épreuve des compromis locaux}

Cette partie s'attache à l'évolution et à la construction des savoirs professionnels intégrant la diversité des points de vue dans des contraintes opérationnelles. Ces savoirs opérationnels participent à la définition des légitimités professionnelles (chap. 9: aménagement ; chap. : 10 équipement urbain ; chap. 11: environnement concerné par des changements d'instrumentation; chap. 12: santé publique). La question des compromis locaux avec les associations est appréhendée et constitue un vecteur de changements cognitifs dans la mesure où des savoirs se constituent progressivement dans les situations d'interaction.

\section{Partie IV - Les légitimités professionnelles à l'épreuve de l'action publique : démarches et recherche}

Ici, il s'agit de contributions théoriques intégrant des grilles d'analyses sociologiques au niveau macro (chap. 13 : axé sur un panorama historique des relations entre État et professions et chap. 14 : traitant des professions libérales d'avocat engagé), au niveau méso (chap. 15 : décrit le développement des régulations de l'action publique et de la dimension sociétale des groupes professionnels entre autonomie d'action et autonomie de contrôle), au niveau micro (chap. 16: porte sur la légitimité professionnelle et sociale, importante pour l'action publique, en mobilisant des experts dans des cadres procéduraux en développement).

Au total, les modifications de l'action publique interfèrent avec les professions et les organisations concrètes de l'activité professionnelle. La conclusion de l'ouvrage en rappelle les grandes lignes, comme la recherche d'individualisation des services sous la pression des usagers et son corollaire de recherche de mesure de performance. Ces mutations sont occasionnées par l'influence de la construction européenne et se traduisent nationalement. Il en résulte une injonction paradoxale. Cette double contrainte repose, d'une part sur la volonté de mesurer l'efficacité et de mieux hiérarchiser des indicateurs dans des procédures fondées sur des indicateurs, d'autre part une meilleure prise en compte de la diversité des attentes des usagers et des cas d'espèce. Pour les professionnels, ces évolutions se traduisent par une polyvalence fondée sur des actions de coordination et d'animation de réseaux. Les auteurs soulignent en particulier que l'ensemble de ces évolutions aboutit à remettre en cause le modèle néo-corporatiste qui a longtemps été le modèle de la structuration étatique française. 


\section{Référence de l'ouvrage}

Le Bianic T. et Vion A. (sous la dir.) (2008), Action publique et légitimités professionnelles, LGDJ, « Droit et société », avril, 347 p. 